\title{
Direct nitration of five membered heterocycles
}

\author{
Alan R. Katritzky*§ ${ }^{\S}$ Eric F.V. Scriven ${ }^{\S}$, Suman Majumder ${ }^{\S}$, Rena G. Akhmedova ${ }^{\S}$, \\ Novruz G. Akhmedov, ${ }^{\S}$ and Anatoliy V. Vakulenko ${ }^{\S}$ \\ ${ }^{\S}$ Center for Heterocyclic Compounds, University of Florida, Department of Chemistry, \\ Gainesville, Florida 32611-7200, USA \\ E-mail: Katritzky@chem.ufl.edu
}

\section{Dedicated to Dr. A.V. Rama Rao on the occasion of his $70^{\text {th }}$ birthday}

(received 21 Dec 04; accepted 14 Jan 05; published on the web 28 Jan 05)

\begin{abstract}
Direct nitration of a variety of furans, pyrroles, thiophenes, pyrazoles, imidazoles, isoxazoles and thiazoles (17 compounds) with nitric acid/trifluoroacetic anhydride affords mononitro derivatives in average yield of $60 \%$.
\end{abstract}

Keywords: Nitration, nitric acid, trifluoroacetic anhydride, nitrofurans, nitropyrroles, nitrothiophenes, nitropyrazoles, nitroimidazoles, nitroisoxazoles, nitrothiazoles

\section{Introduction}

Nitro derivatives of five-membered heterocycles are of considerable interest: some are biologically active ${ }^{1}$ with anti-inflammatory or vasodilator activity ${ }^{2}$ others are useful synthetic intermediates for many biologically active compounds; for instance, nitroimidazoles form the basis of nitro-heterocycles analogous to megazol, an antiparasitic agent ${ }^{3}$.

Nitration of five membered ring heterocycles like furans ${ }^{4}$, pyrroles $^{5}$, thiophenes ${ }^{6}$, pyrazoles ${ }^{7}$, imidazoles $^{8}$, isoxazoles ${ }^{9}$ and thiazoles ${ }^{10}$ has usually been carried out using either a mixture of concentrated (or fuming) nitric acid and concentrated sulfuric acid, or in some cases with concentrated nitric acid and acetic anhydride (followed by pyridine in case of furans only). The nitration of some of these heterocycles, for example pyrazoles and imidazoles ${ }^{11}$, isoxazoles ${ }^{12,13}$ and isothiazoles ${ }^{12}$ has been studied kinetically. Previous efforts to find milder nitration conditions for direct nitration have included use of cerium (IV) ammonium nitrate ${ }^{14}$, montmorillonite impregnated with bismuth nitrate ${ }^{15}$ and nitrations with dinitrogen pentoxide ${ }^{16,17}$.

In light of our success in the direct nitration of pyridines and pyridine analogs with concentrated nitric acid in trifluoroacetic anhydride, which we believe involves $\mathrm{N}_{2} \mathrm{O}_{5}{ }^{18}$ led us to apply this method to nitration of five-membered heterocycles and we discuss our results here. 
While the present work was in progress, Shackelford and coworkers reported ${ }^{19}$ the use of tetramethylammonium nitrate in triflic anhydride and included results of nitration of aromatics like furans, thiophenes and isoxazoles. Our work complements and significantly extends that of Shackelford group.

\section{Results and Discussion}

\section{Furans}

Typically furans have been nitrated using acetyl nitrate to give addition products, which are subsequently converted on treatment with pyridine into 2 -nitrofurans ${ }^{20,21,22,23}$. We have now achieved the direct nitration of furan itself and a series of its derivatives with nitric acid in trifluoroacetic anhydride (method A as described in the experimental section) (Scheme 1) (Table 1). Compounds 2a-d were characterized spectroscopically (see Experimental).

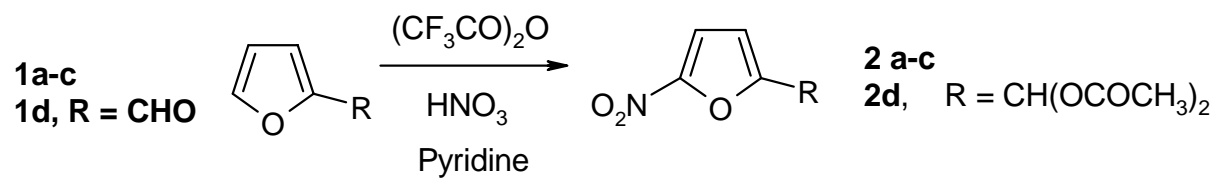

\section{Scheme 1}

Table 1. Nitration of furans

\begin{tabular}{|c|c|c|c|c|c|}
\hline \multirow{2}{*}{ Product } & \multirow[b]{2}{*}{$\mathrm{R}$} & \multirow{2}{*}{$\begin{array}{c}\text { Yield \%, } \\
\text { by method A }\end{array}$} & \multicolumn{3}{|c|}{ Literature methods } \\
\hline & & & $\begin{array}{c}\text { Overall } \\
\text { Yield }^{\mathrm{b}} \% \\
\end{array}$ & $\begin{array}{l}\text { Method }{ }^{\mathrm{e}} / \\
\text { Reagents }\end{array}$ & Ref. \\
\hline \multirow{5}{*}{$2 \mathbf{a}$} & \multirow{5}{*}{$\mathrm{H}$} & \multirow{5}{*}{$68 \%$} & $14 \%$ & ${ }^{\mathrm{e}}\left[\mathrm{NO}_{2}\right]\left[\mathrm{BF}_{4}\right]$ & 21 \\
\hline & & & $\mathrm{c}$ & ${ }^{\mathrm{e}} \mathrm{Ac}_{2} \mathrm{O} ; \mathrm{HNO}_{3}$ & 22 \\
\hline & & & c & ${ }^{\mathrm{e}} \mathrm{Ac}_{2} \mathrm{O} ; \mathrm{HNO}_{3}$ & 23 \\
\hline & & & c & & 24 \\
\hline & & & c & ${ }^{\mathrm{e}}\left[\mathrm{NO}_{2}\right]\left[\mathrm{BF}_{4}\right]$ & 25 \\
\hline \multirow[b]{2}{*}{$2 \mathbf{b}$} & \multirow[b]{2}{*}{$\mathrm{CH}_{3}$} & \multirow[b]{2}{*}{$65 \%$} & c & ${ }^{\mathrm{e}} \mathrm{RaI}_{2} ; \mathrm{Ac}_{2} \mathrm{O}$ & 26 \\
\hline & & & $34 \% \%^{d}$ & $\underset{\mathrm{f}}{\mathrm{HNO}_{3}}$ & 27 \\
\hline \multirow[t]{3}{*}{ 2c } & \multirow[t]{3}{*}{$\mathrm{C}\left(\mathrm{CH}_{3}\right)_{3}$} & \multirow[t]{3}{*}{$75 \%$} & & & novel \\
\hline & & & $40 \%$ & ${ }^{\mathrm{e}} \mathrm{Ac}_{2} \mathrm{O} ; \mathrm{HNO}_{3}$ & 28 \\
\hline & & & c & ${ }^{\mathrm{e}} \mathrm{Ac}_{2} \mathrm{O} ; \mathrm{HNO}_{3}$ & 29 \\
\hline \multirow[t]{3}{*}{$2 d$} & \multirow[t]{3}{*}{$\mathrm{CH}\left(\mathrm{OCOCH}_{3}\right)_{2}$} & \multirow[t]{3}{*}{$58 \%{ }^{\mathrm{a}}$} & c & ${ }^{\mathrm{e}} \mathrm{Ac}_{2} \mathrm{O} ; \mathrm{HNO}_{3}$ & 30 \\
\hline & & & $15 \%$ & ${ }^{\mathrm{e}} \mathrm{Ac}_{2} \mathrm{O} ; \mathrm{HNO}_{3}$ & 31 \\
\hline & & & $43 \%$ & ${ }^{\mathrm{e}} \mathrm{Ac}_{2} \mathrm{O} ; \mathrm{HNO}_{3}$ & 32 \\
\hline
\end{tabular}


Method A is described in the Experimental section; ${ }^{\mathrm{a}}$ using method A but replacing TFAA by $\mathrm{Ac}_{2} \mathrm{O}$; ${ }^{\mathrm{b}}$ overall yield is the final yield after multistep conversion to nitrofurans starting with furan; ${ }^{c}$ cross reference of the compound without reported yield; ${ }^{d}$ overall yield is $34 \%$ following the reaction sequence 2-furfuraldehyde -[80\%] - 2-furfuryl alcohol - [76\%] -2-iodomethyl-5nitrofuran - [56\%]-2-methyl-5-nitrofuran; 'eneral method of synthesis of nitrofurans from furans in two steps using acetyl nitrate via an addition product which is subsequently converted by pyridine into 2-nitrofurans; findirect method starting from 2-iodomethyl-5-nitrofuran using thiophenolate anion as the reagent.

Inspection of Table 1 clearly shows the advantage of our new method. In most published nitration procedures for furan, nitroacetate intermediates had to be isolated. Our one step nitration procedure produces much higher yields without isolation of any intermediate.

\section{Pyrroles}

Again acetyl nitrate has been used for the nitration of pyrrole ${ }^{33}$, to give mainly the 2-nitro derivatives (55\%). Our nitration method B, gave novel compounds 4a-b from 3a-b respectively (Scheme 2), structures were confirmed spectroscopically (see Experimental).

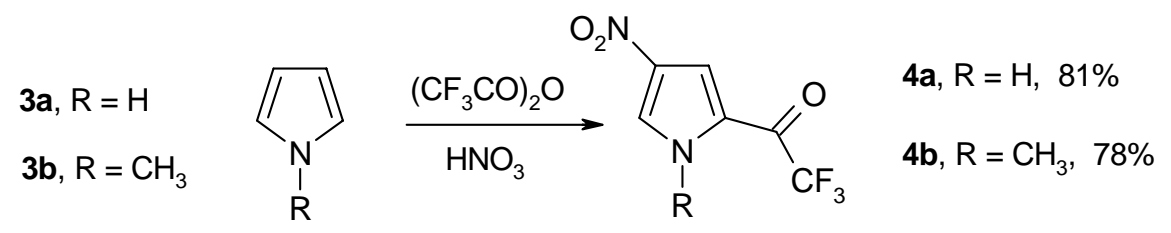

\section{Scheme 2}

\section{Thiophenes}

Thiophenes are easy to nitrate compared to other five membered heterocycles. They react with mild nitrating agents such as copper nitrate ${ }^{34}$, usually in the 2-position. Thiophene (5), on nitration with our reagent gave a 78\% yield of 2-nitroderivative (6) by method B (Scheme 3) (Table 2). Shackelford reported the nitration of methyl 2-thiophenecarboxylate to give a mixture of 2- and 4- nitro derivatives (1.6:1) in 91\% yield ${ }^{19}$.

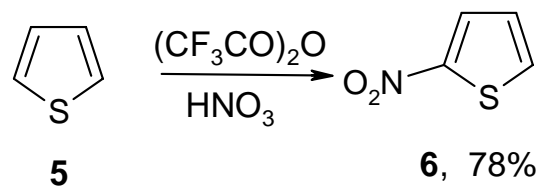

\section{Scheme 3}


Table 2. Nitration of thiophenes

\begin{tabular}{|c|c|c|c|c|}
\hline \multirow[b]{2}{*}{ Product } & \multirow{2}{*}{$\begin{array}{c}\text { Yield \%, } \\
\text { by method B }\end{array}$} & \multicolumn{3}{|c|}{ Literature methods } \\
\hline & & $\begin{array}{l}\text { Overall } \\
\text { Yield \% } \\
\end{array}$ & $\begin{array}{l}\text { Method / } \\
\text { Reagents }\end{array}$ & Ref. \\
\hline \multirow{3}{*}{6 2-nitro } & \multirow{3}{*}{$78 \%$} & $72 \%^{\mathrm{a}}$ & $\mathrm{Cu}\left(\mathrm{NO}_{3}\right)_{2} / \mathrm{Ac}_{2} \mathrm{O}$ & 34 \\
\hline & & $70 \%{ }^{\mathrm{a}}$ & $\mathrm{K} 10$ clay; $\mathrm{HNO}_{3}$ & 35 \\
\hline & & $23 \%^{\mathrm{b}}$ & $\mathrm{NH}_{4} \mathrm{NO}_{3} / \mathrm{Tf}_{2} \mathrm{O}$ & 36 \\
\hline 8 3-bromo-2-nitro & $58 \%$ & $56 \%{ }^{\mathrm{c}}$ & $\mathrm{HNO}_{3}$ & 37 \\
\hline
\end{tabular}

Method: B as described in the Experimental section; ${ }^{a}$ yield from thiophene; ${ }^{b}$ yield from thiophene 2-boronic acid; 'yield from 3-bromothiophene.

We found that the nitration of 3-bromo-thiophene (7) gave a complicated mixture with the main product (8) (58\%). The other component were found to be $\mathbf{9}, \mathbf{1 0 a}-\mathbf{b}$, and $\mathbf{1 1}$. The structure of 10a was not unambigiously diffretiated from the structure 10b (Scheme 4).

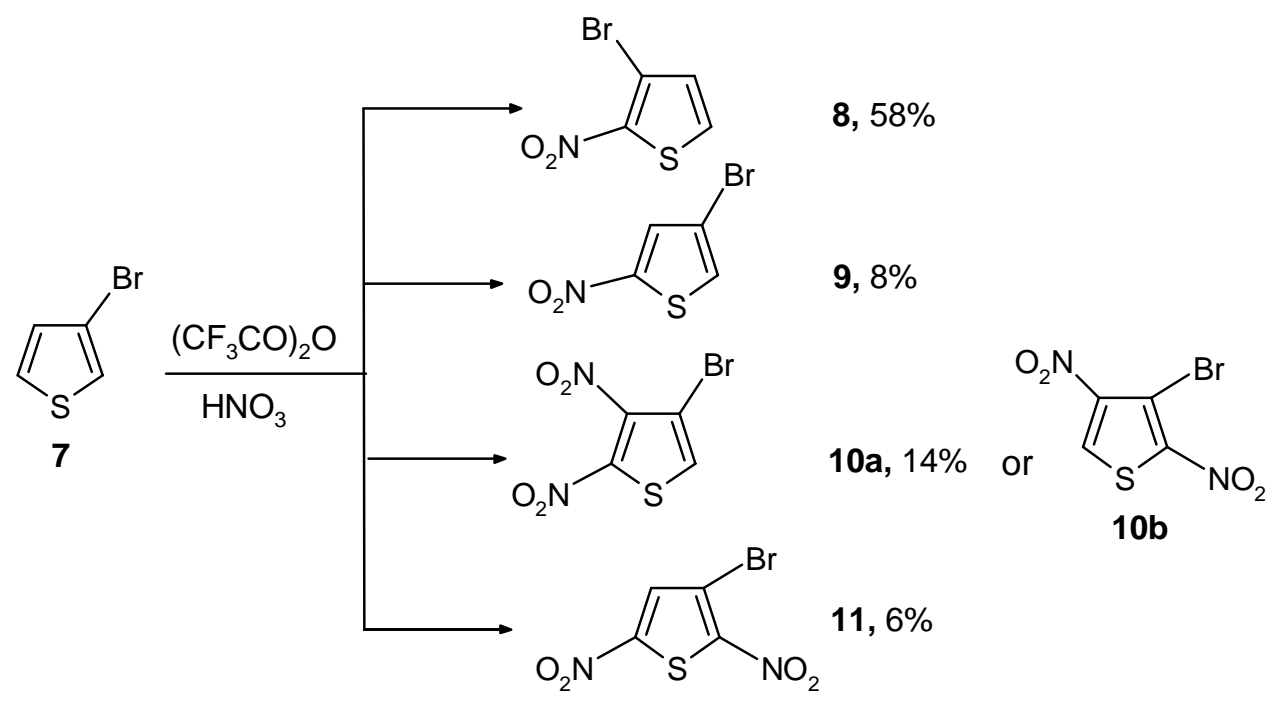

\section{Scheme 4}

\section{Pyrazoles}

Acetyl nitrate has been employed to nitrate pyrazoles at one of the nitrogen atoms and subsequent rearrangement at $140{ }^{\circ} \mathrm{C}$ has been observed to give 3- or 5-nitropyrazoles, sometimes as a mixture. ${ }^{40}$ Pyrazole (12) on treatment with our nitrating system following method $\mathrm{B}$ gave a $41 \%$ yield of the 3,4-dinitrated derivative (13) while $N$-methylpyrazole under the same reaction condition gave a $65 \%$ yield of the 3 -nitro product (13). This orientation was confirmed by nOe experiments (Scheme 5) (Table 3). 


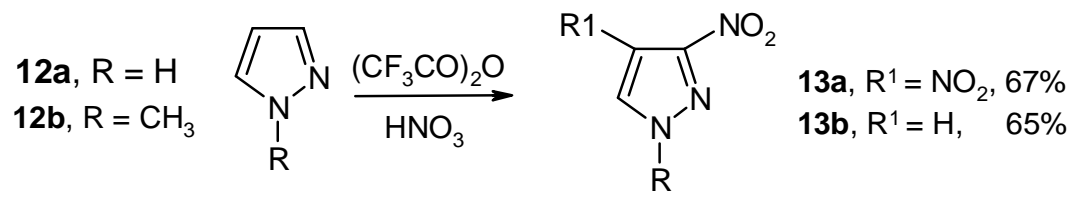

\section{Scheme 5}

Table 3. Nitration of pyrazoles

\begin{tabular}{|c|c|c|c|c|}
\hline \multirow[b]{2}{*}{ Product } & \multirow{2}{*}{$\begin{array}{l}\text { Yield \%, } \\
\text { by method } \\
\text { B }\end{array}$} & \multicolumn{3}{|c|}{ Literature methods } \\
\hline & & $\begin{array}{c}\text { Overall } \\
\text { Yield }^{\text {a }} \%\end{array}$ & $\begin{array}{l}{ }^{\mathrm{f}} \text { Method / } \\
\text { Reagents }\end{array}$ & Ref \\
\hline 13a 3,4-dinitro & $41 \%$ & $68 \%^{\mathrm{b}}$ & 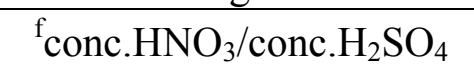 & 40 \\
\hline 13b 3-nitro-1-methyl & $65 \%$ & $\begin{array}{l}28 \%{ }^{\mathrm{c}} \\
3.6 \%\end{array}$ & $\begin{array}{c}{ }^{\mathrm{f}} \text { fuming } \mathrm{HNO}_{3} / 80 \% \text { aq. } \\
\mathrm{H}_{2} \mathrm{SO}_{4} ; 18 \mathrm{hrs} \\
{ }^{\mathrm{f}} \mathrm{HNO}_{3}\end{array}$ & $\begin{array}{l}41 \\
42\end{array}$ \\
\hline 14 3,5-dimethyl-4-nitro & $76 \%$ & $\begin{array}{l}49 \%{ }^{d} \\
\quad e \\
27 \%\end{array}$ & $\begin{array}{c}\mathrm{f}_{70} \% \mathrm{HNO}_{3} \text { in } 80 \% \mathrm{H}_{2} \mathrm{SO}_{4} \\
118^{\circ} \mathrm{C} / 5 \mathrm{hrs} \\
{ }_{\mathrm{f}}^{\mathrm{fNO}} \mathrm{H}_{3} \\
\mathrm{~g}\end{array}$ & $\begin{array}{l}43 \\
44 \\
45\end{array}$ \\
\hline
\end{tabular}

Method B as described in the Experimental section; ${ }^{a}$ overall yield is the final yield after multistep conversion to nitropyrazole starting with pyrazole as starting material; ${ }^{b}$ overall yield is $68 \%$ following the reaction sequence as pyrazole - $[80 \%]-3$-nitropyrazole - $[86 \%]-3,4-$ dinitropyrazole; ' ${ }^{c}$ overall yield is $28 \%$ following the reaction sequence as pyrazole - $[90 \%]-1$ methylpyrazole - [32\%]-3,4-dinitropyrazole; d overall yield is $53 \%$ following the reaction sequence as 3,5-dimethylpyrazole -[94\%] - 4-bromo-3,5-dimethylpyrazole - [53\%]-3,5dimethyl-4-nitropyrazole; ${ }^{\mathrm{e}}$ cross reference of the compound without reported yield; ${ }^{\mathrm{f}}$ direct conversion of pyrazole or its derivatives to nitropyrazole; ${ }^{g}$ ring cyclization to pyrazole using dinitromethane as one of the reactants.

3, 5-Dimethylpyrazole (14), on the other hand, gives only 3,5-dimethyl-4-nitropyrazole in 76\% yield (Scheme 6) (Table 3).

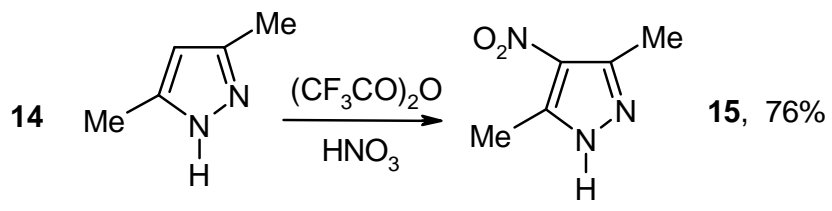

\section{Scheme 6}




\section{Imidazoles}

Imidazoles unsubstituted at nitrogen are easily nitrated by mixed acid nitration ${ }^{46,47}$. The direct nitration of $\mathrm{N}$-substituted imidazoles is more difficult and most nitro- $N$-methylimidazoles have been prepared by the $\mathrm{N}$-methylation of the corresponding nitroimidazoles.

A mixture of 4-nitro- (17a) and 5-nitroimidazoles (17b) was obtained by the action of concentrated nitric acid on 1-methylimidazole (16) in trifluoroacetic anhydride at $0-5^{\circ} \mathrm{C}$ for 12.0 $\mathrm{h}$ according to method B (Scheme 7) (Table 4). Yields quoted for (17) and (19) are that of pure isomers isolated by column chromatography.

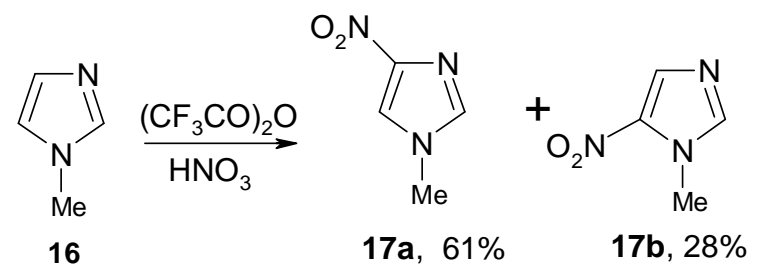

\section{Scheme 7}

Similarly, a mixture of 1,2-dimethyl-4-nitroimidazole (19a) and 1,2-dimethyl-5nitroimidazoles (19b) was obtained by the action of concentrated nitric acid on 1,2dimethylimidazole (18) in trifluoroacetic anhydride at $0-5^{\circ} \mathrm{C}$ for $12.0 \mathrm{~h}$ according to method $\mathrm{B}$ (Scheme 8).

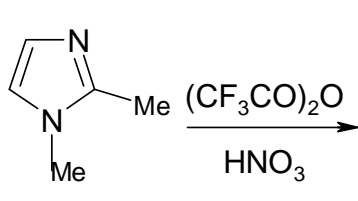

18

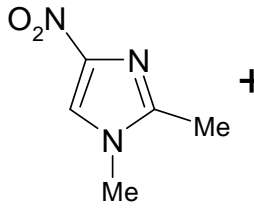

$19 a, 58 \%$<smiles>Cc1ncc([N+](=O)[O-])n1C</smiles>

19b, $35 \%$

\section{Scheme 8}


Table 4. Nitration of imidazoles

\begin{tabular}{|c|c|c|c|c|}
\hline \multirow[b]{2}{*}{ Product } & \multirow{2}{*}{$\begin{array}{l}\text { Yield \%, by } \\
\text { method B }\end{array}$} & \multicolumn{3}{|c|}{ Literature methods } \\
\hline & & $\begin{array}{c}\text { Overall } \\
\text { Yield }^{\text {a }} \%\end{array}$ & $\begin{array}{l}{ }^{\mathrm{f}} \text { Method / } \\
\text { Reagent }\end{array}$ & Ref. \\
\hline 17a 1-methyl-4-nitro & $61 \%$ & $\begin{array}{l}22 \%{ }^{\mathrm{b}} \\
44 \%^{\mathrm{c}}\end{array}$ & $\begin{array}{c}{ }^{\mathrm{f}} \mathrm{Me}_{2} \mathrm{SO}_{4} / \text { range of catalyst. } \\
{ }^{\mathrm{f}} \mathrm{Me}_{2} \mathrm{SO}_{4}\end{array}$ & $\begin{array}{l}48 \\
49\end{array}$ \\
\hline 17b 1-methyl-5-nitro & $28 \%$ & $\begin{array}{l}34 \% \\
57 \% \\
59 \%\end{array}$ & $\begin{array}{c}{ }^{\mathrm{f}} \mathrm{Me}_{2} \mathrm{SO}_{4} / \text { range of catalyst. } \\
{ }^{\mathrm{f}} \text { Dimethyl carbonate/18- } \\
\text { Crown-6 / } \mathrm{K}_{2} \mathrm{CO}_{3} \\
\mathrm{f}_{\mathrm{t}-\mathrm{BuOK} \text {; Reflux in DMF }}\end{array}$ & $\begin{array}{l}48 \\
50 \\
51\end{array}$ \\
\hline 19a 1,2-dimethyl-4-nitro & $58 \%$ & $\begin{array}{l}47 \% \\
25 \%\end{array}$ & $\begin{array}{c}{ }^{\mathrm{f}} \text { Dimethyl carbonate/18- } \\
\text { Crown-6 / } \mathrm{K}_{2} \mathrm{CO}_{3} \\
{ }^{\mathrm{f}} \mathrm{Me}_{2} \mathrm{SO}_{4}\end{array}$ & $\begin{array}{l}50 \\
52\end{array}$ \\
\hline 19b 1,2-dimethyl-4-nitro & $35 \%$ & $80 \%$ & ${ }^{\mathrm{f}} \mathrm{Me}_{2} \mathrm{SO}_{4}$ & 53 \\
\hline
\end{tabular}

Method B as described in the Experimental section. ${ }^{\text {a }}$ overall yield is the final yield after multistep conversion to nitroimidazole starting with imidazole as starting material. ${ }^{\mathrm{b}}$ overall yield is $22 \%$ following the reaction sequence as imidazole -[91\%] - 4-nitroimidazole - [24\%]-1methyl-4-nitropyrazole. 'overall yield is $44 \%$ following the reaction sequence as imidazole [91\%] - 4-nitroimidazole - [48\%] - 1-methyl-4-nitropyrazole. ${ }^{\mathrm{d}}$ overall yield is $34 \%$ following the reaction sequence as imidazole -[91\%] - 4-nitroimidazole - [37\%]-1-methyl-4nitropyrazole. ${ }^{e}$ overall yield is $47 \%$ following the reaction sequence as 2 -methylimidazole [91\%]-2-methyl-4-nitroimidazole $\quad-\quad$ [52\%]-1,2-dimethyl-4-nitroimidazole. findirect conversion of imidazole to 4-nitro-1-methyl or 4-nitro-1,2-dimethyl imidazole through nitration and subsequent $\mathrm{N}$-methylation of imidazoles.

\section{Isoxazoles}

Nitroisoxazoles have been synthesized using various nitrating agents like nitronium fluoroborate $^{54}$, ammonium nitrate/TFAA ${ }^{55}$ or just nitration with mixed acid $^{56}$. Our nitration method A when applied to nitration of isoxazole (20a), 5-methylisoxazole (20b) and 3,5dimethylisoxazole (20c); 2-nitroisoxazole (21a), 5-methyl-3-nitroisoxazole (21b) and 3,5dimethyl-4-nitroisoxazole (21c) were obtained in the yield of $73 \%, 64 \%$, and $72 \%$ respectively. (Scheme 9) (Table 5). Shackelford ${ }^{19}$ found that 3,5-dimethylisoxazole was converted to the 4nitro derivatives in $96 \%$ isolated yield using tetramethylammonium nitrate in triflic anhydride.

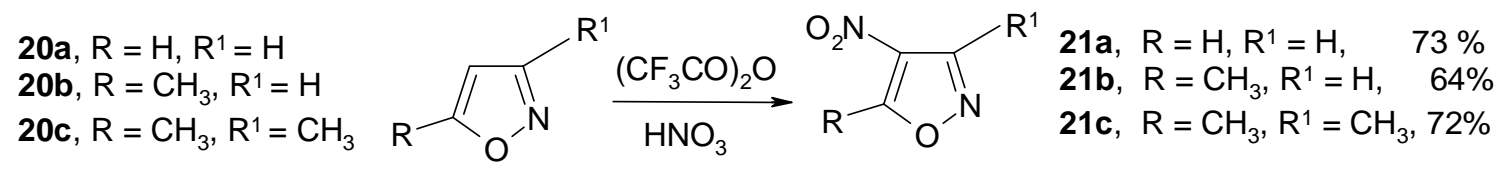

\section{Scheme 9}


Table 5. Nitration of isoxazoles

\begin{tabular}{|c|c|c|c|c|}
\hline \multirow[b]{2}{*}{ Product } & \multirow{2}{*}{$\begin{array}{l}\text { Yield \%, } \\
\text { by method B }\end{array}$} & \multicolumn{3}{|c|}{ Literature methods } \\
\hline & & $\begin{array}{c}\text { Overall } \\
\text { Yield }^{\mathrm{a}} \% \\
\end{array}$ & $\begin{array}{l}{ }^{\mathrm{c}} \text { Method / } \\
\text { Reagent }\end{array}$ & Ref. \\
\hline \multirow{4}{*}{ 21a 4-nitro } & \multirow{4}{*}{$73 \%$} & $51 \%$ & ${ }^{\mathrm{c}} \mathrm{NH}_{4} \mathrm{NO}_{3} / \mathrm{TFA}$ & 57 \\
\hline & & & ${ }^{c} \mathrm{HNO}_{3} / \mathrm{H}_{2} \mathrm{SO}_{4}$ & 58 \\
\hline & & $3.5 \%$ & ${ }^{\mathrm{c}} \mathrm{HNO} 3 / \mathrm{H}_{2} \mathrm{SO}_{4}$ & 56 \\
\hline & & $35 \%$ & ${ }^{\mathrm{c}} \mathrm{NO}_{2} \mathrm{BF}_{4}^{\mathbf{z}}$ & 54 \\
\hline \multirow{3}{*}{ 21b 5-methyl-4-nitro } & \multirow{3}{*}{$64 \%$} & $63 \%$ & ${ }^{\mathrm{c}} \mathrm{NH}_{4} \mathrm{NO}_{3} / \mathrm{TFA}$ & 57 \\
\hline & & $67 \%$ & ${ }^{\mathrm{c}} \mathrm{HNO}_{3} / \mathrm{H}_{2} \mathrm{SO}_{4}$ & 58 \\
\hline & & $80 \%$ & ${ }^{\mathrm{c}} \mathrm{HNO}_{3} / \mathrm{H}_{2} \mathrm{SO}_{4}$ & 55 \\
\hline \multirow{3}{*}{ 21c 3,5-dimethyl-4-nitro } & \multirow{3}{*}{$72 \%$} & $96 \%$ & ${ }^{\mathbf{c}}\left(\mathrm{CH}_{3}\right)_{4} \mathrm{NNO}_{3} / \mathrm{Tf}_{2} \mathrm{O}$ & 19 \\
\hline & & $86 \%$ & ${ }^{\mathrm{c}} \mathrm{HNO} 3 / \mathrm{H}_{2} \mathrm{SO}_{4}$ & 55 \\
\hline & & $20 \%$ & d & 59 \\
\hline
\end{tabular}

Method B as described in the Experimental section. a overall yield is the final yield of the conversion to nitroisoxazole starting with isoxazole as starting material. ${ }^{\mathbf{b}}$ cross reference of the compound without reported yield. ' direct nitration method of isoxazole to nitrooxazole by using different reagents. ${ }^{d}$ ring closure method to synthesize nitrooxazole.

\section{Thiazoles}

Nitration of thiazoles had not previously been studied extensively. ${ }^{60} 2,5$-Dimethylthiazole (22), gave 2,5-dimethyl-4-nitrothiazole (23) in 67\% yield (Scheme 10), which was characterized spectroscopically (see Experimental). We did not study the nitration of thiazole because it was insoluble in our nitration system.

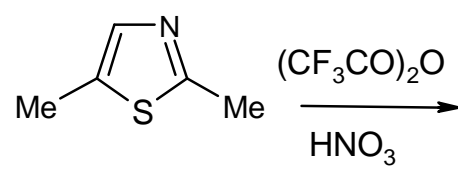

22

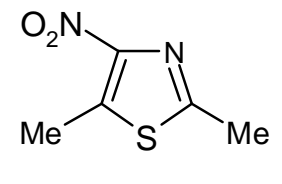

23, $67 \%$

\section{Scheme 10}

\section{Experimental Section}

General Procedures. Melting points are uncorrected. ${ }^{1} \mathrm{H}$ NMR $(300 \mathrm{MHz})$ and ${ }^{13} \mathrm{C}$ NMR $(75$ $\mathrm{MHz}$ ) spectra were recorded in $\mathrm{CDCl}_{3}$ (with TMS for ${ }^{1} \mathrm{H}$ and chloroform- $d$ for ${ }^{13} \mathrm{C}$ as the internal reference) unless specified otherwise. 


\section{General method of preparation of nitro derivatives of five membered heterocycles}

Method A. A mixture of trifluoroacetic anhydride $(10 \mathrm{~mL})$ and fuming nitric acid $(2.4 \mathrm{~mL})$ was chilled at $-15^{\circ} \mathrm{C}$ and after $1 \mathrm{~h}$ a solution of $(10 \mathrm{mmol})$ in trifluoroacetic anhydride $(2 \mathrm{~mL})$ was slowly added to the reaction mixture keeping the temperature at $-15^{\circ} \mathrm{C}$. The reaction mixture was stirred at $-15^{\circ} \mathrm{C}$ for $2 \mathrm{~h}$ and then the solvents were removed and pyridine $(2 \mathrm{~mL})$ was added to the reaction mixture, stirred for $15 \mathrm{~min}$. And then the solvent was again removed and the oily residue was poured in ice and extracted with diethyl ether. The crude product was then purified over a silica gel column to give pure nitro derivatives.

Method B. Trifluoroacetic anhydride $[10 \mathrm{~mL}]$ was chilled in an ice bath and the substrate heterocycle [17 mmol] was slowly added. After $1 \mathrm{~h}$, concentrated nitric acid [3.0 $\mathrm{mL}]$ was added dropwise with cooling. After stirring for $12 \mathrm{~h}$ at room temperature, the excess trifluoroacetic acid and nitric acid were removed under vacuum to get the nitro derivatives, which were purified by column chromatography.

\section{Compound characterization}

2-Nitrofuran (2a). Yellowish microcrystals (68\%), mp 28.0-29.0 ${ }^{\circ} \mathrm{C}$ (lit. ${ }^{61} \mathrm{mp} 28.8-29.2{ }^{\circ} \mathrm{C}$ ). ${ }^{1} \mathrm{H}$ NMR: $\delta 6.68(\mathrm{dd}, J=3.6,1.8 \mathrm{~Hz}, 1 \mathrm{H}), 7.34(\mathrm{dd}, J=3.6,1.0 \mathrm{~Hz}, 1 \mathrm{H}), 7.57(\mathrm{dd}, J=1.8,1.0$ $\mathrm{Hz}, 1 \mathrm{H}) ;{ }^{13} \mathrm{C}$ NMR: $\delta 111.43,113.35,144.95,152.71$.

2-Methyl-5-nitrofuran (2b). White prisms (65\%), mp 42.5-43.5 ${ }^{\circ} \mathrm{C}$ (lit. $\left.{ }^{6} \mathrm{mp} 43.5^{\circ} \mathrm{C}\right) .{ }^{1} \mathrm{H}$ NMR: $\delta 2.46(\mathrm{dd}, J=0.9,0.5 \mathrm{~Hz}, 3 \mathrm{H}), 6.31(\mathrm{dq}, J=3.6,0.9 \mathrm{~Hz}, 1 \mathrm{H}), 7.26(\mathrm{dq}, J=3.6,0.5 \mathrm{~Hz}$, $1 \mathrm{H}) ;{ }^{13} \mathrm{C}$ NMR: $\delta$ 13.98, 110.01, 113.19, 151.26, 156.84 .

2-(tert-Butyl)-5-nitrofuran (2c). Yellowish prisms (75 \%), mp 56.0-57.0 ${ }^{\circ} \mathrm{C} .{ }^{1} \mathrm{H}$ NMR: $\delta 1.36$ (s, $9 \mathrm{H}), 6.24(\mathrm{~d}, J=3.8 \mathrm{~Hz}, 1 \mathrm{H}), 7.23(\mathrm{~d}, J=3.8 \mathrm{~Hz}, 1 \mathrm{H}) ;{ }^{13} \mathrm{C} \mathrm{NMR}: \delta 28.52,33.45,106.39$, 112.75, 151.19, 168.30. Anal. Calcd for $\mathrm{C}_{8} \mathrm{H}_{11} \mathrm{NO}_{3}$ (169.18): C, 56.80; H, 6.55; N, 8.28. Found: C, 56.74; H, 6.75; N, 8.08.

(Acetyloxy)(5-nitro-2-furyl) methyl acetate (2d). White prisms $(58 \%)$, mp 88.6-90.0 ${ }^{\circ} \mathrm{C}$ (lit. $^{30}$ mp 91.0-92.0 $\left.{ }^{\circ} \mathrm{C}\right) .{ }^{1} \mathrm{H}$ NMR: $\delta 2.18(\mathrm{~s}, 6 \mathrm{H}), 6.74(\mathrm{~d}, J=3.7 \mathrm{~Hz}, 1 \mathrm{H}), 7.30(\mathrm{~d}, J=3.7 \mathrm{~Hz}, 1 \mathrm{H})$, $7.72(\mathrm{~s}, 1 \mathrm{H}) ;{ }^{13} \mathrm{C}$ NMR: $\delta$ 20.53, 82.45, 111.45, 112.29, 150.37, 168.05. Anal. Calcd for $\mathrm{C}_{9} \mathrm{H}_{9} \mathrm{NO}_{7}$ (243.17): C, 44.45; H, 3.73; N, 5.76. Found: C, 44.68; H, 3.67; N, 5.68.

2,2,2-Trifluoro-1-(4-nitro-1H-pyrrol-2-yl)-1-ethanone (4a). White prisms (81\%), mp 112.0$113.0{ }^{\circ} \mathrm{C} .{ }^{1} \mathrm{H}$ NMR: $\delta 7.64(\mathrm{q}, J=1.8 \mathrm{~Hz}, 1 \mathrm{H}), 8.48(\mathrm{~d}, J=1.5 \mathrm{~Hz}, 1 \mathrm{H}), 13.86($ br s, $1 \mathrm{H}) ;{ }^{13} \mathrm{C}$ NMR: $\delta 116.14\left(\mathrm{q}, J_{\mathrm{C}-\mathrm{F}}=289.7 \mathrm{~Hz}\right), 114.68\left(\mathrm{q}, J_{\mathrm{C}-\mathrm{F}}=3.4 \mathrm{~Hz}\right), 124.22,129.14,137.76,170.15$ $\left(\mathrm{q}, J_{\mathrm{C}-\mathrm{F}}=36.1 \mathrm{~Hz}\right.$ ). Anal. Calcd for $\mathrm{C}_{6} \mathrm{H}_{3} \mathrm{~F}_{3} \mathrm{~N}_{2} \mathrm{O}_{3}(208.10)$ : C, 34.63; H, 1.45; N, 13.46. Found C, $34.71 ; \mathrm{H}, 1.22 ; \mathrm{N}, 13.26$.

2,2,2-Trifluoro-1-(1-methyl-4-nitro-1H-pyrrol-2-yl)-1-ethanone (4b). White prisms (72 \%), mp 63.5-64.5 ${ }^{\circ} \mathrm{C} .{ }^{1} \mathrm{H}$ NMR: $4.08(\mathrm{~d}, J=0.5 \mathrm{~Hz}, 3 \mathrm{H}), 7.69$ (q, $\left.J=1.8 \mathrm{~Hz}, 1 \mathrm{H}\right), 7.84$ ( dq, $J=1.8$, $0.6 \mathrm{~Hz}, 1 \mathrm{H}) ;{ }^{13} \mathrm{C}$ NMR: $\delta 38.99,116.11,117.29,123.69,131.28,136.07,171.33$. Anal. Calcd for $\mathrm{C}_{7} \mathrm{H}_{5} \mathrm{~F}_{3} \mathrm{~N}_{2} \mathrm{O}_{3}$ (222.12): C, 37.85; H, 2.27; N, 12.61. Found C, 38.01; H, 2.13; N, 12.34.

2-Nitrothiophene (6). White prisms (78\%), mp 42.0-43.0 ${ }^{\circ} \mathrm{C}$ (lit. $\left.{ }^{63} \mathrm{mp} 45.5{ }^{\circ} \mathrm{C}\right) .{ }^{1} \mathrm{H}$ NMR: $\delta$ 7.07 (q, $J=4.1,5.3 \mathrm{~Hz}, 1 \mathrm{H}), 7.55(\mathrm{dd}, J=1.6,5.3 \mathrm{~Hz}, 1 \mathrm{H}), 7.93(\mathrm{dd}, J=1.6,4.1 \mathrm{~Hz}, 1 \mathrm{H}) ;{ }^{13} \mathrm{C}$ 
NMR: $\delta 126.97,128.54,132.50,152.58$. Anal. Calcd for $\mathrm{C}_{4} \mathrm{H}_{3} \mathrm{NO}_{2} \mathrm{~S}$ (129.14): C, 37.20; $\mathrm{H}, 2.34$; N, 10.85. Found C, 37.33; H, 2.22; N, 10.70.

3-Bromo-2-nitrothiophene (8). Yellowish prisms (58\%); mp 79.0-80.0 ${ }^{\circ} \mathrm{C}$ (lit. ${ }^{37} \mathrm{mp} \mathrm{81.0-83.0}{ }^{\circ} \mathrm{C}$ ). ${ }^{1} \mathrm{H}$ NMR: $\delta 7.13(\mathrm{~d}, J=5.6 \mathrm{~Hz}, 1 \mathrm{H}), 7.54(\mathrm{~d}, J=5.6 \mathrm{~Hz}, 1 \mathrm{H}) ;{ }^{13} \mathrm{C}$ NMR: $\delta 112.95,130.97$, 132.54, 146.54. Anal. Calcd for $\mathrm{C}_{4} \mathrm{H}_{2} \mathrm{BrNO}_{2} \mathrm{~S}$ (208.03): C, 23.09; H, 0.97; N, 6.73. Found C, 23.38; H, 0.78; N, 6.53.

3-Bromo-5-nitrothiophene (9). Yellowish prisms (8\%); mp 45.0-46.0 ${ }^{\circ} \mathrm{C}$ (lit. $\left.{ }^{64} \mathrm{mp} 46-47.0{ }^{\circ} \mathrm{C}\right)$. ${ }^{1} \mathrm{H}$ NMR: $\delta 7.47(\mathrm{~d}, J=1.9 \mathrm{~Hz}, 1 \mathrm{H}), 7.85(\mathrm{~d}, J=1.9 \mathrm{~Hz}, 1 \mathrm{H}) ;{ }^{13} \mathrm{C}$ NMR: $\delta 109.98,129.46$, 130.55, 152.06.

3-Bromo-4,5-dinitro-thiophene (10a or 10b). Yellow prisms (4\%); mp 178-180.0 ${ }^{\circ} \mathrm{C}$, lit. ${ }^{65} \mathrm{mp}$ $165-166^{\circ} \mathrm{C}$ for 10b; ${ }^{1} \mathrm{H}$ NMR: $\delta 7.95(\mathrm{~s}, 1 \mathrm{H}) ;{ }^{13} \mathrm{C}$ NMR: $\delta 112.82,131.26,133.17,152.03$.

3-Bromo-2,5-dinitro-thiophene (11). Yellow microcrystals prisms (10\%); m.p.: 111-112 ${ }^{\circ}$, lit. ${ }^{67}$ m.p. : $112-113{ }^{\circ} \mathrm{C} ;{ }^{1} \mathrm{H}$ NMR: $\delta 7.89$ (s, 1H); ${ }^{13} \mathrm{C}$ NMR: $\delta 111.28,131.46,148.40,151.70$.

3,4-Dinitro-1H-pyrazole (13a). White prisms (41\%); mp 90-91 ${ }^{\circ} \mathrm{C}$ (lit. ${ }^{40} \mathrm{mp} 87.5-88.5$ ). ${ }^{1} \mathrm{H}$ NMR: $\delta 8.57$ (s, 1H); ${ }^{13} \mathrm{C}$ NMR: $\delta 132.38,133.58,135.38$.

1-Methyl-3-nitro-1H-pyrazole (13b). White prisms (65\%), mp 81.0-82.0 ${ }^{\circ} \mathrm{C}$ (lit. ${ }^{41} \mathrm{mp} \mathrm{80.0-}$ $\left.84.0{ }^{\circ} \mathrm{C}\right) .{ }^{1} \mathrm{H}$ NMR: $\delta 4.02(\mathrm{~s}, 3 \mathrm{H}), 6.89(\mathrm{~d}, J=2.4 \mathrm{~Hz}, 1 \mathrm{H}), 7.44(\mathrm{dq}, J=2.4,0.3 \mathrm{~Hz}, 1 \mathrm{H}) .{ }^{13} \mathrm{C}$ NMR: $\delta 40.42,103.14,132.64,155.37$. Anal. Calcd for $\mathrm{C}_{4} \mathrm{H}_{5} \mathrm{~N}_{3} \mathrm{O}_{2}$ (127.10): C, 37.80; H, 3.97; N, 33.06. Found C, 38.16; H, 3.79; N, 32.79.

3,5-Dimethyl-4-nitro-1H-pyrazole (15). Brownish needles (76\%), mp 122.0-123.0 ${ }^{\circ} \mathrm{C}$ (lit. ${ }^{43} \mathrm{mp}$ 126.0-127.0 $\left.{ }^{\circ} \mathrm{C}\right) .{ }^{1} \mathrm{H}$ NMR: $\delta 2.46(\mathrm{~s}, 6 \mathrm{H}) ;{ }^{13} \mathrm{C}$ NMR: $\delta 12.69,130.04,143.46$. Anal. Calcd for $\mathrm{C}_{5} \mathrm{H}_{7} \mathrm{~N}_{3} \mathrm{O}_{2}$ (141.13): C, 42.55; H, 5.00; N, 29.77. Found C, 42.67; H, 5.02; N, 29.43.

1-Methyl-4-nitro-1H-imidazole (17a). White prisms (39\%), mp 133.0-134.0 ${ }^{\circ} \mathrm{C}$ (lit. ${ }^{48} \mathrm{mp} 134{ }^{\circ} \mathrm{C}$ ). ${ }^{1} \mathrm{H}$ NMR: $\delta 3.83(\mathrm{~s}, 3 \mathrm{H}), 4.72(\mathrm{br} \mathrm{d}, J=1.5 \mathrm{~Hz}, 1 \mathrm{H}), 7.78(\mathrm{~d}, J=1.5 \mathrm{~Hz}, 1 \mathrm{H}) ;{ }^{13} \mathrm{C} \mathrm{NMR}: \delta 34.55$, 120.19, 136.61, 148.00. Anal. Calcd for $\mathrm{C}_{4} \mathrm{H}_{5} \mathrm{~N}_{3} \mathrm{O}_{2}$ : C, 37.80; H, 3.97; N, 33.06. Found C, 38.16; $\mathrm{H}, 3.83 ; \mathrm{N}, 32.86$.

1-Methyl-5-nitro-1H-imidazole (17b). Orange prisms (22\%), mp 59.0-60.0 ${ }^{\circ} \mathrm{C}\left(\right.$ lit. $\left.{ }^{33} \mathrm{mp} 60.0{ }^{\circ} \mathrm{C}\right)$. ${ }^{1} \mathrm{H}$ NMR: $\delta 4.02$ (d, $\left.J=0.6 \mathrm{~Hz}, 3 \mathrm{H}\right), 7.59$ (br s, $\left.1 \mathrm{H}\right), 7.98(\mathrm{~d}, J=1.1 \mathrm{~Hz}, 1 \mathrm{H}) ;{ }^{13} \mathrm{C}$ NMR: $\delta 35.02$, $132.86,138.87,141.52$.

1,2-Dimethyl-4-nitro-1H-imidazole (19a). White needles $(53 \%), \mathrm{mp} 182.0-184.0{ }^{\circ} \mathrm{C}$ (lit. ${ }^{50} \mathrm{mp}$ $\left.184{ }^{\circ} \mathrm{C}\right) .{ }^{1} \mathrm{H}$ NMR: $\delta 2.39(\mathrm{~s}, 3 \mathrm{H}), 2.47(\mathrm{~s}, 3 \mathrm{H}), 8.54(\mathrm{~s}, 1 \mathrm{H}), 8.87(\mathrm{~s}, 1 \mathrm{H}) ;{ }^{13} \mathrm{C}$ NMR: $\delta .12 .85$, $33.74,120.71,146.10,145.07$.

1,2-Dimethyl-5-nitro-1H-imidazole (19b). White prisms $\left(18 \%\right.$ ), mp 134.0-135.0 ${ }^{\circ} \mathrm{C}$ (lit. ${ }^{66} \mathrm{mp}$ 134.0-135.0 $\left.{ }^{\circ} \mathrm{C}\right) .{ }^{1} \mathrm{H}$ NMR: $\delta 2.39(\mathrm{~s}, 3 \mathrm{H}), 2.47(\mathrm{~s}, 3 \mathrm{H}), 8.54(\mathrm{~s}, 1 \mathrm{H}), 8.87(\mathrm{~s}, 1 \mathrm{H}) ;{ }^{13} \mathrm{C}$ NMR: $\delta$. 13.70, 32.90, 131.89, 149.98 .

4-Nitroisoxazole (21a). Yellow prisms (73\%), mp 45.0-46.0 ${ }^{\circ} \mathrm{C}$ (lit. $\left.{ }^{57} \mathrm{mp} 46.0-47.0{ }^{\circ} \mathrm{C}\right) .{ }^{1} \mathrm{H}$ NMR: $\delta 8.85(\mathrm{~s}, 1 \mathrm{H}), 9.32(\mathrm{~s}, 1 \mathrm{H}) ;{ }^{13} \mathrm{C}$ NMR: $\delta$ 144.44, 157.84. Anal. Calcd for $\mathrm{C}_{3} \mathrm{H}_{2} \mathrm{~N}_{2} \mathrm{O}_{3}$ (114.06): C, 31.59; H, 1.77; N, 24.56. Found C, 31.63; H, 1.55; N, 24.31.

5-Methyl-4-nitroisoxazole (21b). Yellow oil (64 \%), (lit. ${ }^{57}$ bp 88.0-90.0 / 18 Torr). ${ }^{1} \mathrm{H}$ NMR: $\delta$ $2.87(\mathrm{~d}, J=0.7 \mathrm{~Hz}, 3 \mathrm{H}), 8.76(\mathrm{q}, J=0.7 \mathrm{~Hz}, 1 \mathrm{H}) ;{ }^{13} \mathrm{C} \mathrm{NMR}: \delta 12.97,131.08,145.88,170.66$. 
Anal. Calcd for $\mathrm{C}_{4} \mathrm{H}_{4} \mathrm{~N}_{2} \mathrm{O}_{3}$ (128.09): C, 37.51; H, 3.15; N, 21.87. Found C, 37.68; H, 2.95; N, 21.64

3,5-Dimethyl-4-nitroisoxazole (21c). Yellowish prisms (72\%), mp 63.0-64.0 ${ }^{\circ} \mathrm{C}$ (lit. ${ }^{55} \mathrm{mp} 63.0$ $\left.64.0{ }^{\circ} \mathrm{C}\right) .{ }^{1} \mathrm{H}$ NMR: $\delta 2.56(\mathrm{~s}, 3 \mathrm{H}), 2.82$ (s, 3H); ${ }^{13} \mathrm{C}$ NMR: $\delta 11.49,13.81,130.14,155.50$, 171.89 .

2,5-Dimethyl-4-nitro-1,3-thiazole (23). Brownish prisms $(67 \%)$, mp 55.5-56.5 ${ }^{\circ} \mathrm{C}$ (lit. ${ }^{60} \mathrm{mp}$ $\left.56.5{ }^{\circ} \mathrm{C}\right) .{ }^{1} \mathrm{H}$ NMR: $\delta 2.71(\mathrm{~s}, 3 \mathrm{H}), 2.79(\mathrm{~s}, 3 \mathrm{H}) ;{ }^{13} \mathrm{C} \mathrm{NMR}: \delta 13.10,19.08,138.36,150.84$, 161.29. Anal. Calcd for $\mathrm{C}_{5} \mathrm{H}_{6} \mathrm{~N}_{2} \mathrm{O}_{2} \mathrm{~S}$ (158.18): C, 37.97; H, 3.82; N, 17.71. Found C, 38.08; H, $3.74 ; \mathrm{N}, 17.54$.

\section{References and Notes}

1. Cherkofsky, S. C. US Pat. 4199 592, 1980; Chem. Abstr. 1980, 93, 186352.

2. Kusumi, T.; Nakanishi, K. US Pat. 4288 445, 1981; Chem. Abstr. 1981, 95, 209675 e.

3. Chauvière, G.; Bouteille, B.; Enanga, B.; de Albuquerque, C.; Croft, S. L.; Dumas, M.; Périé, J. J. Med. Chem. 2003, 46, 427.

4. Heaney, H.; Ahn, J. S. In Comprehensive Heterocyclic Chemistry II; Katritzky, A. R.; Rees, C. W.; Scriven, E. F. V. Eds., Pergamon Press: New York, 1996; Vol. 2, p 300.

5. Black, D. St. C. In Comprehensive Heterocyclic Chemistry II; Katritzky, A. R.; Rees, C. W.; Scriven, E. F. V. Eds. Pergamon Press: New York, 1996; Vol. 2, p 43.

6. Rajappa, S.; Natekar, M. V. In Comprehensive Heterocyclic Chemistry II; Katritzky, A. R.; Rees, C. W.; Scriven, E. F. V. Eds., Pergamon Press: New York, 1996; Vol. 2, p 502.

7. Elguero, J. In Comprehensive Heterocyclic Chemistry II, Katritzky, A. R.; Rees, C. W.; Scriven, E. F. V. Eds., Pergamon Press: New York, 1996; Vol. 3, p 36.

8. Grimmett, M. R. In Comprehensive Heterocyclic Chemistry II, Katritzky, A. R.; Rees, C. W.; Scriven, E. F. V. Eds., Pergamon Press: New York, 1996; Vol. 3, p 119.

9. Sutharchanadevi, M.; Murugan, R. In Comprehensive Heterocyclic Chemistry II, Katritzky, A. R.; Rees, C. W.; Scriven, E. F. V. Eds., Pergamon Press: New York, 1996; Vol. 3, p 237.

10. Dondoni, A.; Merino, P. In Comprehensive Heterocyclic Chemistry II, Katritzky, A. R.; Rees, C. W.; Scriven, E. F. V. Eds., Pergamon Press: New York, 1996; Vol. 3, p 389.

11. Austin, M. W.; Blackborow, J. R.; Ridd, J. H.; Smith, B. V. J. Chem. Soc. 1965, 1051.

12. Burton, A. G.; Forsythe, P. P.; Johnson, C. D.; Katritzky, A. R. J. Chem. Soc. (B) 1971, 2365.

13. Katritzky, A. R.; Konya, M.; Tarhan, H. O.; Burton, A. G. J. Chem. Soc., Perkin Trans. 2 1975, 1627.

14. Sathunuru, R.; Rao, U. N.; Biehl, E. ARKIVOC 2003, (xv), 124.

15. Samajdar, S.; Becker, F. F.; Banik, B. K. ARKIVOC 2001, (viii), 27.

16. Bakke, J. M. Pure Appl. Chem. 2003, 75, 1403.

17. Bakke, J. M.; Gautun, H. S. H.; Rømming, C; Sletvold, I. ARKIVOC 2001, (x), 26. 
18. Katritzky, A. R.; Scriven, E. F.V.; Majumder S.; Akhmedova, R.G.; Vakulenko, A. V.; Akhmedov, N. G.; Murugan R.; Abboud, K. A. Org. Biomol. Chem. 2005, 3, 538.

19. Shackelford, S. A.; Anderson, M. B.; Christie, L. C.; Goetzen, T.; Guzman, M. C.; Hananel, M. A.; Kornreich, W. D.; Li, H.; Pathak, V. P.; Rabinovich, A. K.; Rajapakse, R. J.; Truesdale, L. K.; Tsank, S. M.; Vazir, H. N. J. Org. Chem. 2003, 68, 267.

20. Gilman, H.; Young, R. V. J. Am. Chem. Soc. 1933, 56, 464.

21. Oláh, G.; Kuhn, S.; Mlinkó, A. J. Chem. Soc. 1956, 4257.

22. Kolb, V. M.; Darling, S. D.; Koster, D. F.; Meyers, C. Y. J. Org. Chem. 1984, 49, 1636.

23. Balina, G.; Kesler, P.; Petre, J.; Pham, D.; Vollmar, A. J. Org. Chem. 1986, 51, 3811.

24. Venters, K; Trusule, M Latvijas PSR Zinatnu Akademijas Vestis, Kimijas Serija 1977, 2, 230, Chem. Abst. 1977, 87, 68045.

25. Lukevics, E.; Ignatovich, L.; Goldberg, Y.; Polyak, F.; Gaukhman, A.; Rozite, S.; Popelis, J. J. Organomet. Chem. 1988, 11.

26. Symons, M. C. R.; Bowman, W. R. J. Chem. Soc., Perkin Trans. 2 1987, 1133.

27. Beadle, C. D.; Bowman, W. R.; Prousek, J. Tetrahedron. Lett. 1984, 25, 4979.

28. Gilman, H.; Wright, G. F. J. Am. Chem. Soc. 1930, 52, 2550.

29. Vlaovic, D.; Milic, B. L.; Mackenzie, K. J. Chem. Res. (S) 1989, 156.

30. Venters, K. K.; Trushule, M. A.; Kumerov, G. F. Khim.-Farm. Zh. 1989, 23, 1506.

31. Lolya, D. O.; Venter, K. K.; Liepin'sh, É. É.; Trushule, M. A.; Giller, S. A. Chem. Heterocycl. Compd. (Engl. Transl.) 1976, 502.

32. Witte, M.; Lind, C. J. US Pat. 2,502, 114, 1950, Chem. Abstr. 1950, 44, 27525.

33. Morgan, K. J.; Morrey, D. P. Tetrahedron 1966, 22, 57.

34. Putokhin, N. I. Sbornik. Nauch. Trudov Kuibyshev. Ind. Inst. im. V. V. Kuibysheva 1955, 271, Chem. Abstr. 1957, 51, 90630.

35. Choudary, B. M.; Kantam, M. L.; Ramprasad, K. V.; Ranganath, K. V. S. Eur. Pat. 1346 991, 2003; Chem. Abstr. 2003, 139, 261163.

36. Salzbrunn, S.; Simon, J.; Prakash, G. K. S.; Petasis, N. A.; Olah, G. A. Synlett 2000, 1485.

37. Astrakhantseva, N. I.; Zhiryakov, V. G.; Abramenko, P. I. Chem. Heterocycl. Compd. (Engl. Transl.) 1976, 1123.

38. Spinelli, D.; Guanti, G.; Dell'Erba, C. J. Chem. Soc., Perkin Trans. 2 1972, 441.

39. Steinkopf, W.; Jacob, H.; Penz, H. Liebigs Annalen 1934, 512, 136.

40. Janssen, J. W. A. M.; Koeners, H. J.; Kruse, C. G.; Habraken, C. L. J. Org. Chem. 1973, 38, 1777.

41. Grimmett, M. R.; Lim, K. H. R. Aust. J. Chem. 1978, 31, 689.

42. Vasilevskii, S. F.; Shvartsberg, M. S. Izv. Akad. Nauk SSSR, Ser. Khim. (Engl. Transl.) 1980, 778.

43. Chang, K-C.; Grimmett, M. R.; Ward, D. D.; Weavers, R. T. Aust. J. Chem. 1979, 32, 1727.

44. Svistunova, I. V.; Shapkin, N. P.; Nikolaeva, O. V. Zh. Obshch. Khim. 2002, 72, 962 (Russ.). 
45. Laikhter, A. L.; Cherkasova, T. I.; Semenov, V. V. Izv. Akad. Nauk SSSR, Ser. Khim. (Engl. Transl.) 1991, 2463.

46. Erb, B.; Rigo, B.; Pirotte, B.; Couturier, D. J. Heterocycl. Chem. 2002, 39, 15.

47. Suzuki, H.; Nonoyama, N. J. Chem. Res.(S) 1996, 244.

48. Dehmlow, E. V.; Richter, R.; Zhivich, A. B. J. Chem. Res. (S) 1993, 504.

49. Horváth, A. Synthesis 1995, 1183.

50. Lissel, M. Liebigs Ann. Chem. 1987, 77.

51. Bergman, J.; Sand, P. Tetrahedron. Lett. 1984, 25, 1957.

52. Dockner, T.; Frank, A.; Riewe, G.; Wetzler, M. Ger. Offen. 3132 025, 1983; Chem. Abstr. 1983, 98, 198227.

53. Bonnamas, C.; Massonneau, V.; Mulhauser, M.; Rouy, N. Eur. Pat. Appl. 325 512, 1990, Chem. Abstr. 1990, 112, 77185.

54. Kusumi, T.; Nakanishi, K. US Pat. 4288445 1981; Chem. Abstr. 1981, 95, 209675.

55. Pérez, J. D.; Wunderlin, D. A. J. Org. Chem. 1987, 52, 3637.

56. Kochetkov, N. K.; Khomutova, E. D. J. Gen. Chem.(Engl. Transl.) 1959, 29, 533.

57. Reiter, L. A. J. Org. Chem. 1987, 52, 2714.

58. Pascual, A. Helv. Chim. Acta 1989, 72, 556.

59. Fruttero, R.; Calvino, R.; Ferrarotti, B.; Gasco, A.; Aime, S.; Gobetto, R.; Chiari, G.; Calestani, G. J. Chem. Soc., Perkin Trans. 2 1987, 523.

60. Friedmann, A.; Bouin, D.; Metzger, J. Bull. Soc. Chim. Fr. 1970, 3155.

61. Freure, B. T.; Johnson, J. R. J. Am. Chem. Soc. 1931, 53, 1142.

62. Rinkes, I. J. Recl. Trav. Chim. Pays-Bas. 1930, 49, 1118.

63. Babasinian, V. S. Org. Synth. 1934, 14, 76.

64. Motoyama, R.; Nishimura, S.; Imoto, E.; Murakami, Y.; Hari, K.; Ogawa, J. Nippon Kagaku Zasshi 1957, 78, 954; Chem. Abstr. 1960, 54, 74585.

65. Gronowitz, S.; Dahlgren, K. Ark. Kemi 1963, 21, 201; Chem. Abstr. 1963, 59, 69005.

66. Bowman, W. R.; Crosby, D.; Westlake, P. J. J. Chem. Soc., Perkin Trans. 2 1991, 73. 